\title{
Resonant absorption of a chemically sensitive layer based on waveguide gratings
}

\author{
Laurent Davoine,,$^{1, \star}$ Vincent Paeder, ${ }^{2}$ Guillaume Basset, ${ }^{1}$ Marc Schnieper, ${ }^{1}$ \\ and Hans Peter Herzig ${ }^{2}$ \\ ${ }^{1}$ Thin Film Optics Department, Centre Suisse d'Electronique et de Microtechnique (CSEM), \\ Tramstrasse 99, Muttenz $\mathrm{CH}-4132$, Switzerland \\ 2Optics \& Photonics Technology Laboratory, Ecole Polytechnique Fédérale de Lausanne (EPFL), \\ Rue A.-L. Breguet 2, Neuchâtel CH-2000, Switzerland \\ *Corresponding author: davoine.laurent@gmail.com
}

Received 8 August 2012; revised 5 November 2012; accepted 3 December 2012; posted 4 December 2012 (Doc. ID 173986); published 11 January 2013

\begin{abstract}
A colorimetric sensor providing a direct visual indication of chemical contamination was developed. The sensor is a combination of a chemically sensitive dye layer and a resonant waveguide grating. Enhancement of the light absorption by the photonic structure can be clearly seen. The detection is based on the color change of the reflected light after exposure to a gas or a liquid. Low-cost fabrication and compatibility with environments where electricity cannot be used make this device very attractive for applications in hospitals, industries, with explosives, and in traffic. () 2013 Optical Society of America OCIS codes: $\quad 130.2790,310.2785$
\end{abstract}

\section{Introduction}

Chemical and biochemical sensors are becoming of major interest in everyday life, in particular visual sensors that could warn people in real time of any chemical attack or atmospheric pollution. Different chemical systems are on the market that change their color when exposed to external chemical agents either through air or if immersed in liquids. The best known is the litmus paper that changes color irreversibly depending on the $\mathrm{pH}$ of the liquid it is immersed in. Current time-temperature indicators, which are used on packages of sensitive goods, such as food or chemicals to determine if the package content is still safe to use or has exceeded its lifetime, depend on the dynamics of a tailored chemical reaction to achieve a visual color change. Flexible temperature sensors use liquid crystals to achieve reversible, visible color changes at very specific temperatures.

$1559-128 X / 13 / 030340-10 \$ 15.00 / 0$

(C) 2013 Optical Society of America
The major drawbacks of these purely chemical approaches are their poor sensitivity and the significant chemical engineering required to achieve small changes in their optical appearance. Combining physical and chemical interactions brings enhanced sensitivity and easier functional tailoring. In this paper we propose a visual gas chemical sensor that combines a chemical sensing layer with a resonant waveguide grating. The latter consists of a subwavelength grating that incorporates a high refractive index thin-film acting as a waveguide layer.

Resonances of such optical structures are called guide mode resonances [1,2]. Under specific illumination conditions (wavelength, angle), the grating couples light by first order diffraction into a waveguide mode. By reciprocity, the mode is resonantly re-emitted in the incident medium, producing sharp reflection peak and transmission dip. These sharp spectral features have been used to produce photonic devices, such as narrowband filters, polarizers, laser mirrors, tunable filters, and security devices [3-5]. Such mode resonances are highly sensitive to the geometrical and material parameters of the 
waveguide structures, which make them also suitable for biosensing applications [6-9].

In this manuscript, we use the resonance of a waveguide grating to emphasize the change in absorption of a chemically sensitive dye layer after gas contamination. We show that light absorption within a thin layer can be strongly amplified by the resonance of a guided mode.

To demonstrate the feasibility of a visual gas sensor based on resonant waveguide grating and dye thin film, we show the efficacy of the sensor at detecting ammonia vapors. Optimization routines based on rigorous simulations have been implemented to select the best suited photonic structure. The visibility of color changes by the human eye can be expressed by color difference quantity (DE) values (see Section 3.G, "Color Changes"). Therefore DE values are extracted from the reflected spectra to evaluate the color changes after ammonia exposure.

\section{Working Principle of the Visual Gas Sensor Based on Resonant Waveguide Gratings}

The device configuration is depicted in Fig. 1. A broad spectrum light source illuminates the structure at a specific angle. The user observes the device in reflection. At resonant wavelengths, the light coupled into the structure interacts strongly with the absorbing dye. The resonance peaks are attenuated as a function of the dye absorption coefficient.

By choosing carefully the position of the reflection peaks within the visible spectrum, the dye absorption produces a strong color change.

\section{A. Resonant Absorption with Waveguide Gratings}

One of the key points to achieve resonant absorption relies on the optimization of the coupling between the dye thin film and the waveguiding structure. The amount of absorbed light depends on the local field amplitude, the intrinsic absorption of the material (dye thin film) itself, and on the volume interaction. Therefore, high quality factor resonances (small resonant bandwidth) are required to provide high

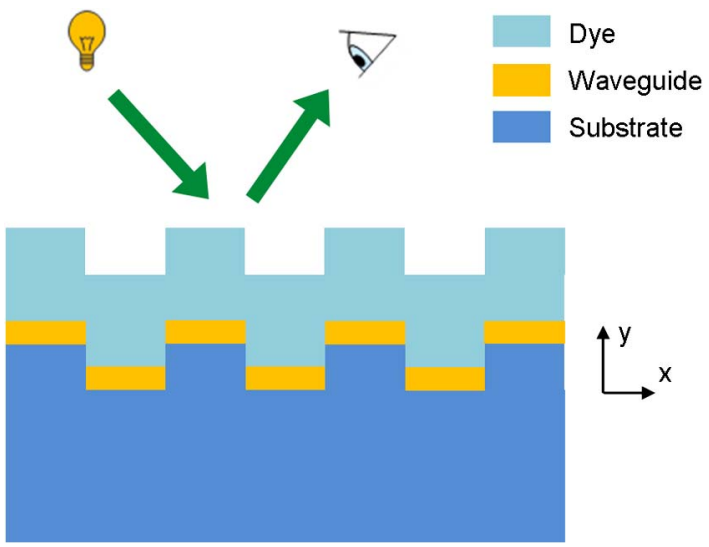

Fig. 1. (Color online) Sketch of the resonant waveguide structure with a dye thin film deposited on top of the structure. The upcoming light couples inside the structure, propagates along the surface, and eventually decouples out of the structure. local field amplitude and high propagation distance inside the waveguide.

Without considering any scattering or absorption losses, the quality factor of the resonance describes the leakage of the mode to the external media. It has been shown that the absorption losses of the material have to match the leakage rate of the mode in order to have resonant absorption through an impedance matching condition [10-13]. The photon lifetime $\tau_{o}$, related to the optical losses (related to the quality factor), needs to be equal to the photon lifetime $\tau_{a}$, related to the absorption losses. The quality factor $Q_{o}$ and the absorption coefficient can be expressed in terms of $\tau_{o}$ and $\tau_{a}$, respectively:

$$
\begin{gathered}
Q=\varpi \tau_{o} \\
\alpha=\frac{1}{\tau_{a} \frac{c}{n}},
\end{gathered}
$$

where $\omega$ is the light pulsation, $c$ is the light speed in vacuum, and $n$ is the refractive index of the material.

The so-called impedance matching condition $\tau_{o}=$ $\tau_{a}$ leads to the following relation:

$$
Q=\frac{2 \pi n}{\alpha \lambda} .
$$

The absorption coefficient is related to the extinction coefficient by the following formula:

$$
\alpha=\frac{4 \pi k}{\lambda} \text {. }
$$

Finally, the quality factor can be expressed as

$$
Q=\frac{n}{2 k}
$$

Therefore, the optimal extinction is obtained by choosing the resonance quality factor as a function of the absorption coefficient of the dye thin film. Materials with high absorption coefficients require a low quality factor structure, exhibiting large resonant bandwidth and a poor absorption enhancement. On the contrary, materials with low absorption coefficients require high quality factor structure, leading to a small resonant bandwidth and to a large absorption enhancement.

\section{B. Simplified Absorption Model}

For the waveguiding structure to work as a sensing device, the key feature to know is the ability to decrease the resonant peaks during resonant process. To answer this question, we only need to know the maximum achievable absorption, assuming that the phase matching condition (resonance condition) is still valid and there is no transmission $(A=$ $1-R-T)$. Consequently we have developed in this section a simple model to predict the maximum absorption. 
Let's consider an external plane wave impinging the structure at resonance conditions (wavelength, incidence angle). Because of the resonant regime, we assume the whole incoming light is trapped inside the waveguide layer as guided waves. Since the waveguide is periodically modulated, the guided modes are scattered out. Thus, they are called resonant leaky modes [14]. A leaky mode can be described by its constant complex propagation along the structure ( $x$ direction):

$$
\beta=\beta_{r}+j \beta_{i},
$$

where $\beta_{r}$ denotes the real propagation constant and $\beta_{i}$ the attenuation constant of the leaky wave amplitude. The envelope of the mode along the structure can be expressed as follows:

$$
P(x)=P_{o} \exp \left(-2 \beta_{i} x\right)
$$

where $P_{o}$ is the initial power in the waveguide.

If any absorbing material is present inside the leaky structure, an absorption coefficient can be added to the attenuation constant of the leaky wave. Therefore the power remaining inside the leaky mode is now

$$
P(x)=P_{o} \exp \left[-2\left(\beta_{i}+\alpha\right) x\right]
$$

The integration of this power function over the distance gives the amount of light that is extracted or absorbed from the leaky mode. The difference between the case of a leaky mode without and with an absorption coefficient represents the amount of light that is actually absorbed by the structure with an absorption coefficient, as shown in Fig. $\underline{2}$.

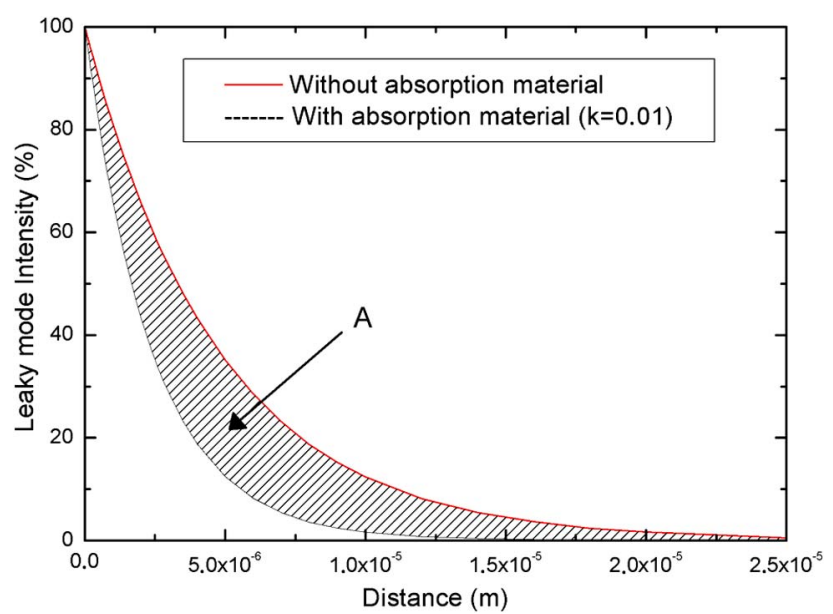

Fig. 2. (Color online) Power remaining inside the leaky mode according to the propagation distance. Red curve corresponds to a leaky mode without any absorption material. Blue curve corresponds to the same leaky mode resonance but with an absorption material. Area A between the red and the blue curve represents the amount of light that is actually absorbed by the structure having an extinction coefficient of $k=0.01$.
Therefore, we subtract the integration of these two intensity functions along $x$ to get $A$, the amount of light absorbed by the structure:

$$
\begin{aligned}
& A=\int_{0}^{+\infty} P_{0} e^{\left(-2 \beta_{i} x\right)} \mathrm{d} x-\int_{0}^{+\infty} P_{0} e^{\left(-2\left(\beta_{i}+\alpha\right) x\right)} \mathrm{d} x \\
& A=P_{0} \frac{\alpha}{2 \beta_{i}\left(\beta_{i}+\alpha\right)} .
\end{aligned}
$$

We normalize $A$ to get the portion of light absorbed in comparison to the initial amount of light (area under curve 1):

$$
\begin{aligned}
& A_{\text {Rel }}=\frac{\frac{P_{0} \alpha}{2 \beta_{i}\left(\beta_{i}+\alpha\right)}}{\frac{P_{0}}{2 \beta_{i}}} \\
& A_{\text {Rel }}=\frac{\alpha}{\left(\beta_{i}+\alpha\right)} .
\end{aligned}
$$

The damping distance $\beta_{i}$ can be expressed in terms of the photon lifetime related to the resonance:

$$
\beta_{i}=\frac{1}{\tau_{0} \frac{c}{n}} .
$$

Recalling $\left(\alpha=\left(1 / \tau_{a}(c / n)\right)\right)$, the matching condition $\tau_{0}=\tau_{a}$ implies $\beta_{i}=\alpha$; thus,

$$
A_{\text {Rel }}=0.5 \text {. }
$$

Therefore one can predict that half of the incident light can be absorbed in the resonant regime when impedance matching condition is valid. Note that we suppose all along the demonstration that the entire mode is inside the absorption material. Therefore $A_{\mathrm{Rel}}$ would correspond to the maximum achievable absorption under impedance matching condition.

\section{Light Absorption Enhancement According to the Quality Factor of the Resonance}

From the above result we can determine the light enhancement provided by the resonant waveguide structure. We define the enhancement factor $G$ by the ratio of the light absorbed with the photonic structure to the light absorbed by a thin layer in transmission (Beer-Lamberts' law). In the best case, half of the light is absorbed by the photonic structure, which yields

$$
G=\frac{0.5}{1-e^{(-\alpha l)}} .
$$

$\alpha$ is related to the quality factor of the resonance through the matching conditions described above [Eq. ()] ]. Therefore the enhancement factor can be expressed as a function of the quality factor:

$$
G=\frac{0.5}{\left(1-e^{\left(-\frac{2 \pi n l}{Q \lambda}\right)}\right)} .
$$




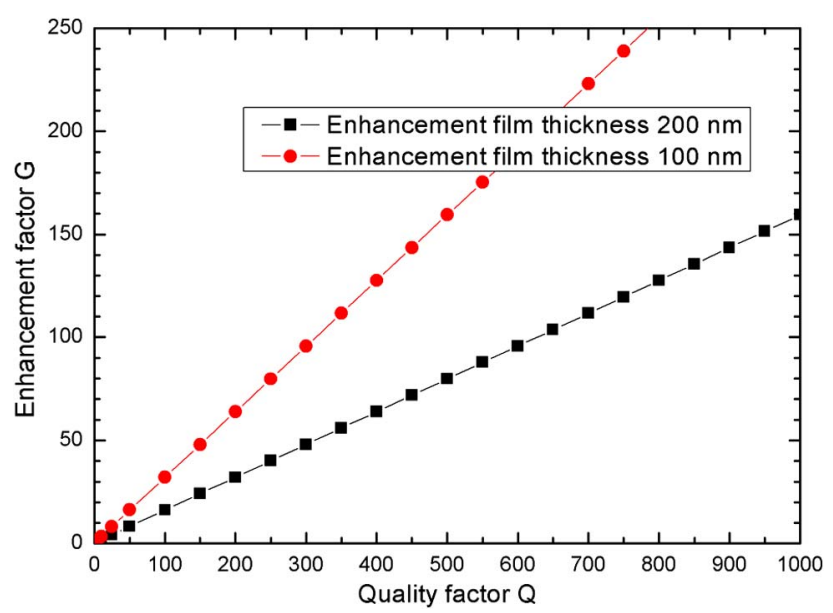

Fig. 3. (Color online) Light enhancement factor $G$ at impedance matching conditions according to the quality factor of the waveguide grating. Wavelength of $600 \mathrm{~nm}$ and refractive index of 1.5 are assumed.

In Fig. 3 the enhancement factor $G$ for film thicknesses $10 \overline{0}$ and $200 \mathrm{~nm}$ and $n=1.5, \lambda=600 \mathrm{~nm}$ is presented.

\section{Validation of the Absorption Model with Rigorous Simulations}

To demonstrate the validity of our model, rigorous calculations have been carried out using rigorous coupled-wave analysis [15]. The structure under study is shown in Fig. 4. The overlap of the mode with the absorbing dye is optimized with an evolutionary algorithm [16]. The free parameter space includes the following parameters: grating period, grating depth, waveguide thickness dye thickness, and resonance wavelength. Two structures corresponding to different target resonance bandwidths have been selected. Their optical and geometrical parameters are detailed in Fig. $\underline{4}$ and Table $\underline{1}$.

Figure 5 shows the reflection $(0 R)$, the transmission $(0 T)$, and the absorption (Abs $=1-0 R-0 T$ ) of the dye thin film at the resonant wavelength and as a function of the extinction coefficient $(k)$. It shows that the absorption increases with $k$ up to an

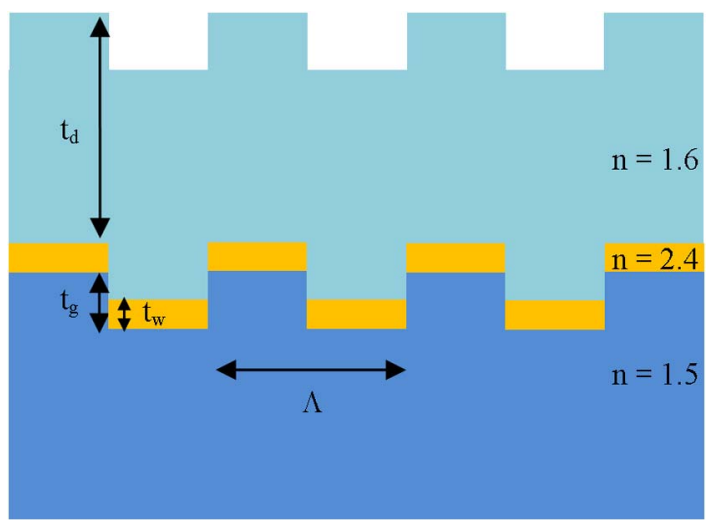

Fig. 4. (Color online) Waveguide grating structure with optical and geometric parameters.
Table 1. Optical and Geometrical Parameters of Two Resonant Waveguide Structures

\begin{tabular}{lcc}
\hline Parameters & Structure 1 & Structure 2 \\
\hline$\Lambda$, grating period $(\mathrm{nm})$ & 310 & 320 \\
$t_{g}$, grating depth $(\mathrm{nm})$ & 50 & 50 \\
$t_{w}$, waveguide thickness $(\mathrm{nm})$ & 24 & 13 \\
$t_{d}$, dye thickness $(\mathrm{nm})$ & 200 & 200 \\
$\theta$, incidence angle $\left(^{\circ}\right)$ & 20 & 20 \\
$\lambda_{r}, r$ Resonant wavelength $(\mathrm{nm})$ & 599.0 & 600.1 \\
$\Delta \lambda$, resonance bandwidth $(\mathrm{nm})$ & 1.25 & 0.60 \\
$Q$, Quality factor & 479 & 1000 \\
$n_{\text {eff }}$, effective mode index & 1.62 & 1.55 \\
$k$ (impedance matching conditions) & $1.69 \mathrm{E}-3$ & $7.7 \mathrm{E}-4$ \\
\hline
\end{tabular}

optimum. Transmission $0 T$ increases concurrently as the phase matching conditions, required to have constructive interferences in reflection, cease to be valid. We can notice that the optimum absorption of structure 2 is obtained at a lower extinction coefficient than structure 1 .

(a) Structure 1

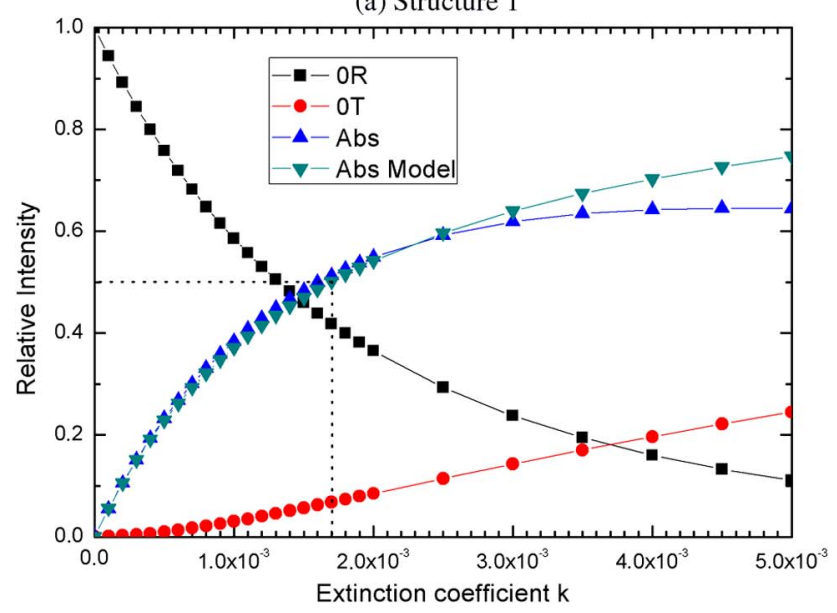

(b) Structure 2

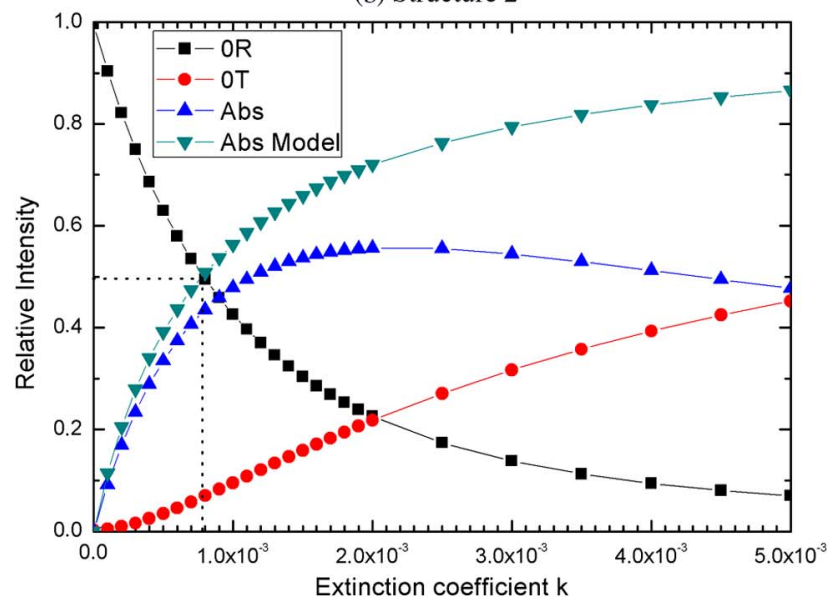

Fig. 5. (Color online) Rigorous simulations of the reflection (0R), transmission $(0 \mathrm{~T})$, and absorption (Abs $=1-0 R-0 T$ ) of the dye thin film at the resonant wavelength as a function of the extinction coefficient $(k)$. Graph (a) corresponds to structure 1 and graph (b) to structure 2 (see Table 1). Absorption calculated with the simplified absorption model is also shown (Abs Model). Dashed lines indicate the coordinates of the impedance matching conditions. 
We can notice from the rigorous simulations that absorption is about 0.5 at impedance matching conditions for both structures as predicted by the relations derived above. The absorption model curves match very well the absorption obtained from the rigorous simulations up to the impedance matching conditions for structure 1 , contrary to structure 2 . In the latter case the transmission increases rapidly with $k$, which indicates that the resonance conditions no longer hold. Note that reflection peaks can be attenuated by more than $50 \%$ with higher absorption coefficients. One deviates in this case from the impedance matching condition and the resonant light (sum of reflection and absorption) decreases to the profit of transmission. In our case, we are interested in reducing reflection peaks with a minimum variation of the absorption coefficient, as it corresponds to low gas concentration detection. Consequently, the highest enhancement factor $G$ is required. The enhancement factors of the two structures according to the extinction coefficient are derived from the rigorous simulations, as depicted in Fig. 6. The enhancement is higher for low $k$ values and becomes weaker for higher extinction coefficient. This demonstrates that the resonant absorption regime yields a stronger damping of the reflection peaks.

\section{E. Considerations for Visual Sensors}

In the case of a visual sensor, the naked eye is the final element of the sensor chain. Therefore the resolution of our sensor corresponds to the smallest change that can be detected by the naked eye. We propose to design a structure exhibiting two reflection peaks varying differently with the absorption change of the dye layer. Changing the ratio of these two peaks brings variation in the three color attributes (hue, saturation and lightness). Thus, the color differences are expected to be higher than in the case of one reflection peak, which relies only on the lightness changes. Substantial color changes can be obtained by carefully choosing their position over

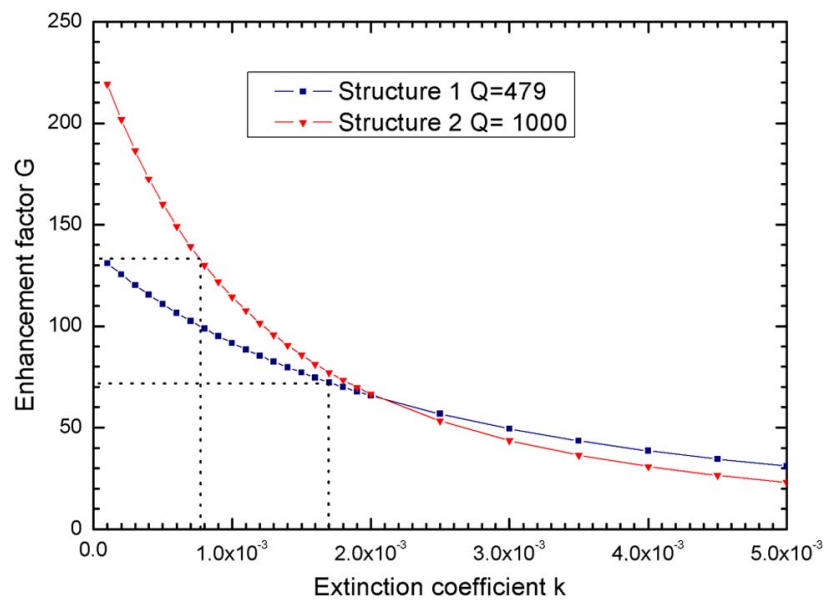

Fig. 6. (Color online) Light enhancement factor $G$ retrieved from rigorous simulations of structure 1 and structure 2 . the visible spectrum. In addition, since the naked eye sees a broad spectrum (from 380 to $780 \mathrm{~nm}$ ) a thin resonant peak has a very low impact on the resulting color. Therefore the level of reflection out of the resonance dictates the minimum width of the resonant reflection peak. Anti-reflection structures have to be integrated in the waveguide grating design in order to exhibit lower side band levels.

\section{Gaseous Ammonia Sensing with Waveguide Gratings}

To show the feasibility of a visual gas sensor based on resonant waveguide grating and dye thin films, we demonstrate the efficacy of the sensor configuration depicted in Fig. 1 at detecting ammonia vapors. The sensitive layer is composed of a mixture of bromocresol purple (BCP) and polymethylmethacrylate (PMMA), providing a host matrix to the dye molecules. BCP has been previously used in an absorptionbased optical sensor [17]. PMMA can easily be dissolved in a number of commercial solvents and mixed with various indicator dyes. The absorption of the dye changes after exposition to ammonia.

\section{A. Sensor Fabrication}

The sensor fabrication process is based on standard techniques suitable for low-cost mass production. The fabrication of the waveguide grating can be divided into two steps: fabrication of the grating and deposition of the waveguide layer. The subwavelength grating is fabricated by a common stamping process. A master grating made of fused silica is fabricated by laser holography followed by reactive ion etching. A sol-gel material (Ormocer, from Micro Resist Technology) is dispensed onto a 2 in square glass plate. A nickel shim is obtained from the master grating by electroplating and is used to replicate the grating into the sol-gel by UV-casting process $[18,19]$. In a second step, a zinc sulfide (ZnS) layer is evaporated to obtain the waveguide layer. A solution of BCP $[1 \%(\mathrm{w} / \mathrm{v})]$ and PMMA $[3 \%(\mathrm{w} / \mathrm{v})]$ is spin coated on top of the waveguide structure to provide the chemically sensitive layer.

\section{B. Experimental Setup}

The experimental setup to measure reflection (and transmission) spectra under dry air and ammonia atmosphere is shown in Fig. 7. The light source consists

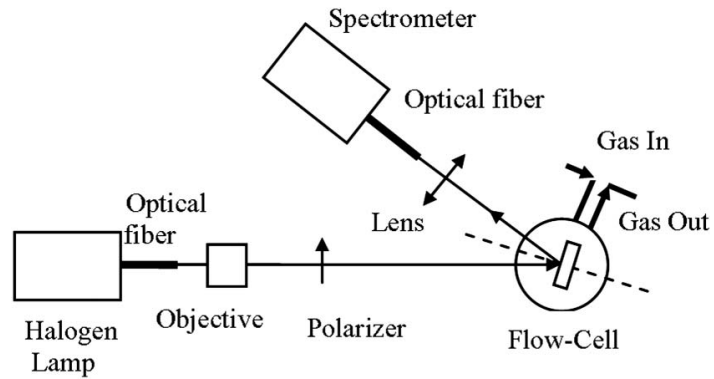

Fig. 7. Experimental setup to measure reflection spectrum under dry air and ammonia atmosphere. 
of a Halogen lamp (DH-2000) from Mikropack connected to an optical fiber from Ocean optics (400 $\mu \mathrm{m}$ core diameter). The light coming out of the fiber is collimated with a microscope objective and illuminates the front side of the sensor. The light is polarized with a Glan Thomson polarizer to select the TE mode (electric field parallel to the grating lines). A lens collects and focuses the reflected light onto another optical fiber connected to a spectrometer (HR4000 CG-UV-NIR) from Ocean Optics. The sensor is placed into a large flow-cell to allow measurements under different ammonia gas concentrations.

\section{Sensor Design}

The aim of the present visual gas sensor is to produce a visual color change with a minimum variation of the BCP film absorption, corresponding to a low ammonia concentration exposure. Our approach consists of having two resonant reflection peaks. Different colors can be obtained depending on the relative level of each peak as long as the rest of the reflection spectrum is low. One of the two resonant peaks is positioned on the absorption band of the BCP film when exposed to ammonia. The strategy to obtain an effective absorption of this resonant peak has been explained above. First, it requires a maximum overlap between the waveguide mode profile and the dye thin film. Second, one must carefully choose a quality factor of the resonance in accordance with the absorption coefficient of the dye thin film.

The real and imaginary parts of the refractive index of the BCP layer have been estimated by reflectance and transmission spectroscopy measurements, respectively. These measurements were carried out under dry air and ammonia atmosphere on a BCP film spin-coated on a glass substrate.

\section{Sensor Response to $\mathrm{NH} 3$}

Figure 8 represents transmission measurements of a $\mathrm{BCP}^{-}$film deposited on glass substrate under ammonia atmosphere (100 ppm) according to time

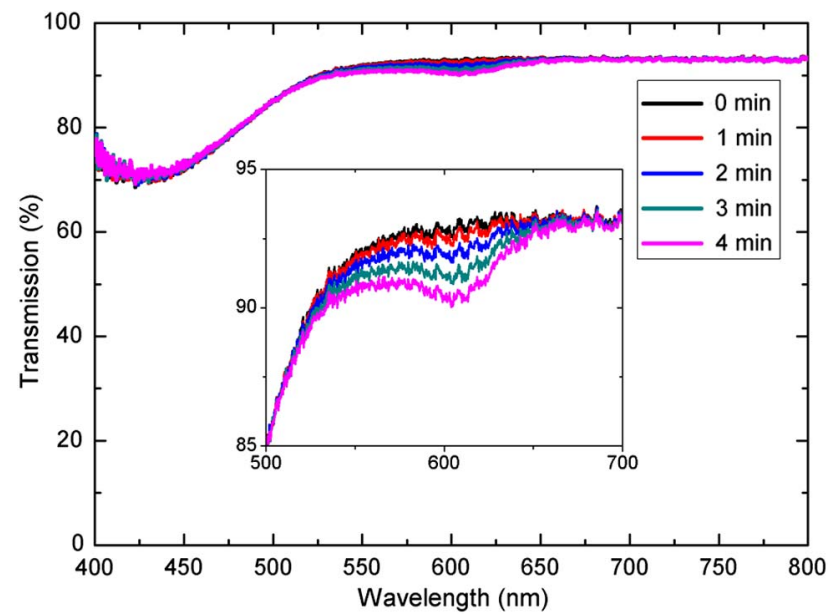

Fig. 8. (Color online) Transmission of BCP film on glass under ammonia exposure (100 ppm) according to time exposure. exposure. Before ammonia exposure there is strong absorption band in the short wavelengths, with a maximum absorption peak at $430 \mathrm{~nm}$. An absorption band appears in the wavelength range of 500 $650 \mathrm{~nm}$ under ammonia atmosphere. The absorption band in the short wavelength slightly decreases under ammonia exposure. Time diffusion of the gas inside the dye thin film is responsible for the strong time dependent response.

The change of absorption of the BCP film on glass is rather low for an ammonia concentration of $100 \mathrm{ppm}$. We believe that much higher sensitivity of the BCP film (same absorption change for a lower ammonia concentration) can be achieved with additional material improvement (gas diffusion, polymer properties, and dye concentration). However, the focus of the present article is to enhance a very low response of the dye (absorption change) that would not be detected by the naked eye without the resonant waveguide structure.

The absorption coefficient $(\alpha)$ and the extinction coefficient $(k)$ of the BCP film have been retrieved from the transmission spectrum by computing the Beer-Lambert's law:

$$
\begin{aligned}
& \alpha=-\frac{\ln (T)}{l} \\
& \alpha=\frac{4 \pi k}{\lambda} \\
& k=\frac{-\ln (T) * \lambda}{4 \pi * l},
\end{aligned}
$$

where $T$ is the transmission, $l$ the thickness of the film, and $\lambda$ the wavelength of light. A reflectivity of $6 \%$ has been considered for this calculation due to the film reflection. The calculated $k$ values according to the wavelength are represented inside Fig. 9 .

The absorption coefficient of the material dictates the quality factor to use to get maximum absorption through the impedance matching condition described in the previous section [Eq. (5)]. Assuming $k=0.01$ at $600 \mathrm{~nm}$ with a mode index of 1.6 we found $Q=80$. Therefore a bandwidth of $7.5 \mathrm{~nm}$ for a resonant peak at $600 \mathrm{~nm}$ has to be selected for the resonant waveguide structure in such case.

\section{E. Numerical Optimization with Rigorous Simulations}

The design of the resonant waveguide grating sensor with BCP film is based on the following requirements:

- low reflection off-resonance

- impedance matching conditions, dictating the maximum resonant bandwidth (minimum quality factor $Q$ )

- resonant peak around $600 \mathrm{~nm}$ matching the absorption band of BCP under ammonia atmosphere 


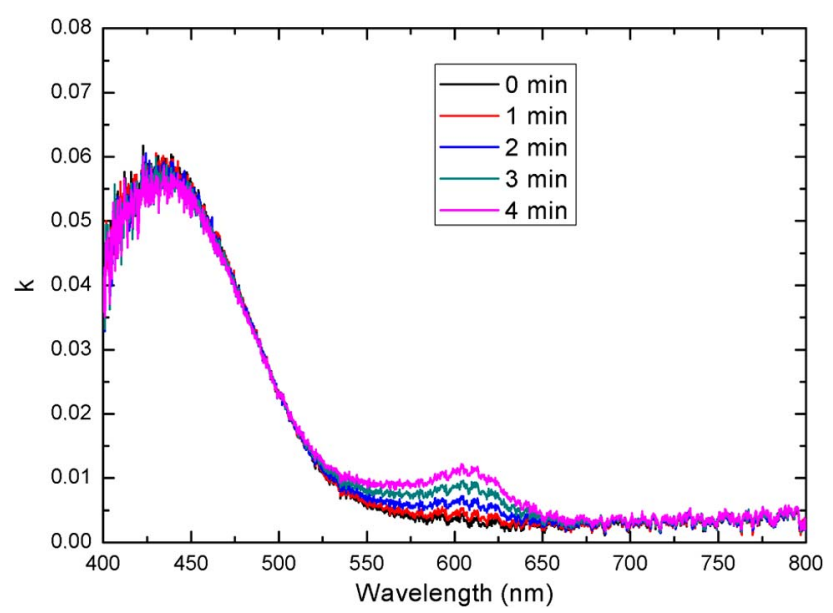

Fig. 9. (Color online) Extinction coefficient $(k)$ of the BCP film under ammonia exposure (100 ppm), retrieved from the transmission measurement.

To meet all the above requirements a numerical optimization routine based on rigorous simulations has been implemented by adjusting the waveguide structure parameters (grating period, grating depth, waveguide thickness, dye thickness, and incidence angle). The optical and geometrical parameters of the optimized structure are detailed in Table 2 .

Figure 10 shows reflection spectrum of the optimized structure calculated with rigorous simulations. A comparison between the case where the dye thin film has an extinction coefficient $k$ of 0 and 0.01 (constant over wavelength) is presented. We can notice that the resonant peak at $580 \mathrm{~nm}$ is much more affected by the absorption than the other peak at $440 \mathrm{~nm}$. Figure 11 shows the evolution of absorption according to extinction coefficient of the dye thin film. Absorption enhancement at the position of the resonant peak is clearly shown. Note the saturation of the absorption for $k$ higher than 0.05 .

\section{F. Reflection Measurements}

The optimized structure was fabricated according to the process described above. Reflection measurements of this structure under ammonia exposure are shown in Fig. 12. There is a good agreement on the position of resonant peaks between calculations and measurements. However, resonant peaks do not yield $100 \%$ efficiency in the measurement spectrum due to scattering losses. In addition, the resonant peak width is smaller since the grating profile is not perfectly square but has a rather sinusoidal shape.

Table 2. Optimized Waveguide Structure Parameters

\begin{tabular}{lc}
\hline Parameters & Optimized Structure \\
\hline$\Lambda$, grating period $(\mathrm{nm})$ & 295 \\
$t_{g}$, grating depth $(\mathrm{nm})$ & 70 \\
$t_{w}$, waveguide thickness $(\mathrm{nm})$ & 50 \\
$t_{d}$, dye thickness $(\mathrm{nm})$ & 160 \\
$\theta$, incidence angle $\left(^{\circ}\right)$ & 17 \\
$\lambda_{r}$, Resonant wavelength $(\mathrm{nm})$ & 582 \\
\hline
\end{tabular}

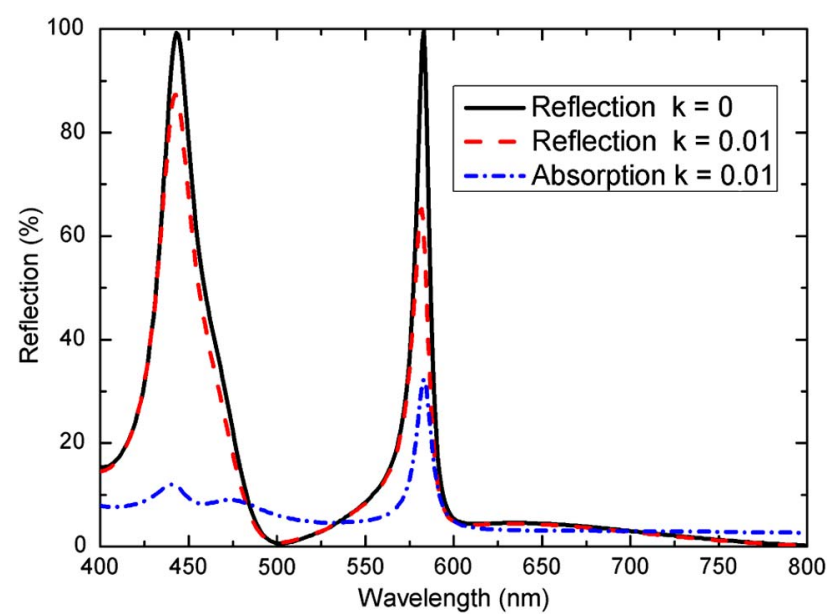

Fig. 10. (Color online) Reflection spectrum of the calculated optimum structure. Black curve: Dye thin film has an extinction coefficient $k$ of 0 . Red and blue curves: Dye thin film has an extinction coefficient $k$ of 0.01 .

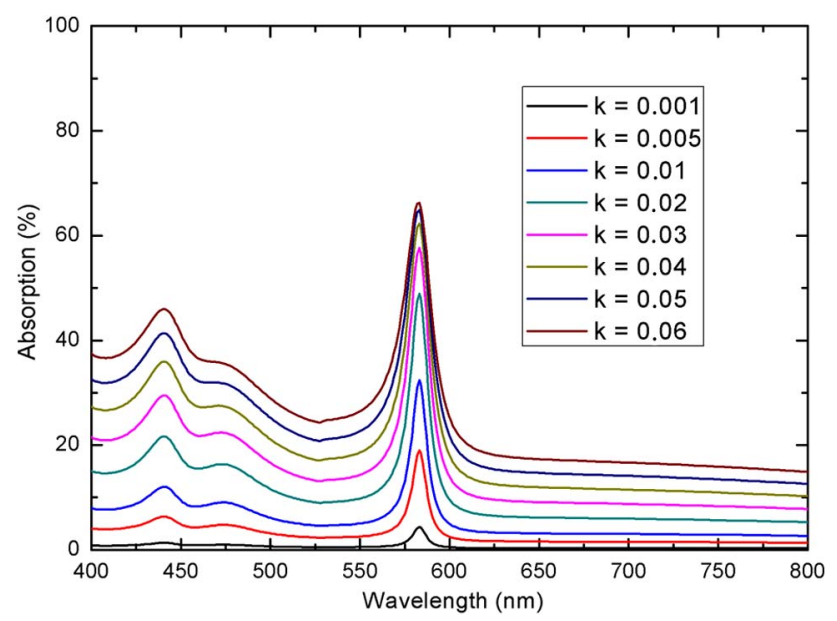

Fig. 11. (Color online) Absorption calculated for the optimized structure for different values of the extinction coefficient $k$ (constant over wavelength) of the dye thin film.

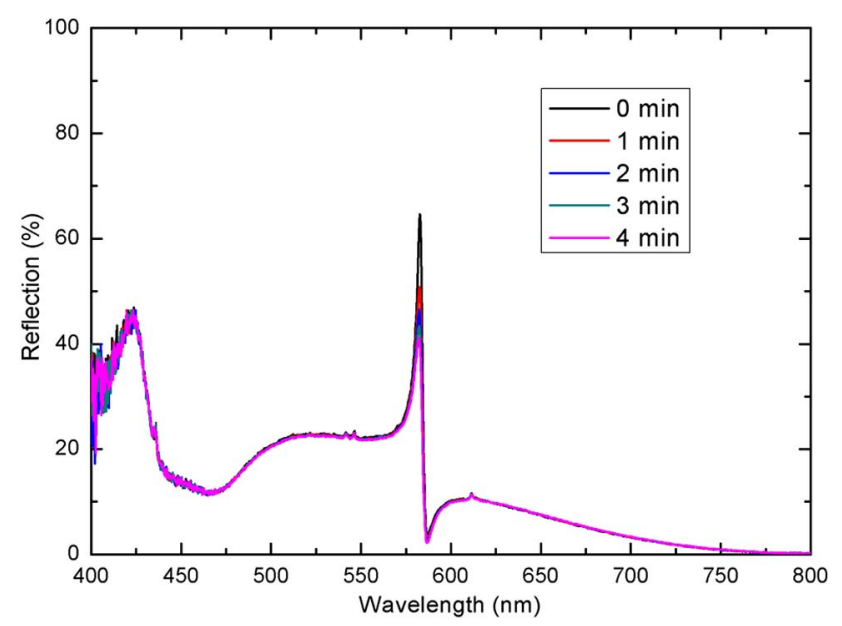

Fig. 12. (Color online) Reflection spectrum of BCP film on waveguide gratings under dry air (blue curve) and ammonia exposure (red curve). 


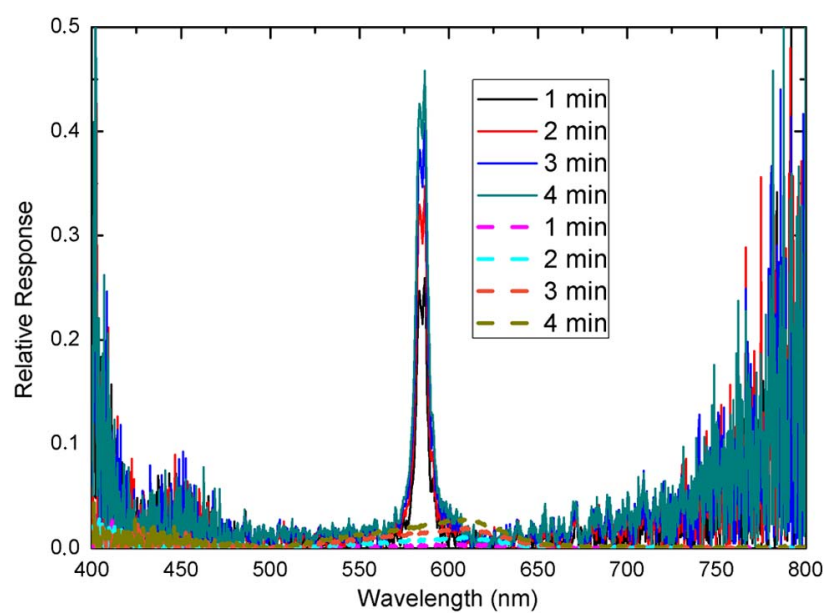

Fig. 13. (Color online) Relative response $\left(\left[\mathrm{R}_{\text {initial }}-R\right] / \mathrm{R}_{\text {initial }}\right)$ of BCP films on glass (dash curves) and on waveguide grating (solid curves) under ammonia exposure (100 ppm).

Since the structure is not symmetric (different refractive indices of the cladding and the substrate), the resonance has an asymmetric line shape [4]. There is a strong attenuation of the peak situated at around $580 \mathrm{~nm}$ under ammonia exposure while the rest of the spectrum remains unchanged.

In order to show the impact of the waveguide grating on the amount of light absorbed, a comparison between the relative response of $\mathrm{BCP}$ film on glass and the relative response of $\mathrm{BCP}$ film on waveguide grating is shown in Fig. 13. It shows a clear enhancement of the response with the waveguide grating structure at the positions of the resonant peaks, which is due to the increase of resonant light absorbed along the dye thin film.

\section{G. Color Changes}

As the present sensor is meant to be visual, the final response is more appropriately represented by figures that define the amount of color changes rather than fluctuations of reflection peaks. The difference between two colors can be calculated using an equation developed by the Commission Internationale de l'Eclairage (CIE) in 1976 called DE. This equation calculates the linear (Euclidian) distance between two points in the Lab 3D space. The color coordinates and the DE have been retrieved from the reflection and transmission spectra measurements. Table 3 shows the color differences for BCP on resonant waveguide gratings and Table $\underline{4}$ for $\mathrm{BCP}$ on glass. It is known that a $\mathrm{DE}$ value

Table 3. Color Coordinates of the Reflection Spectra According to Time Exposure in Ammonia Atmosphere for BCP Film on Waveguide Grating

\begin{tabular}{lccrccc}
\hline Exposure Time $(\min )$ & $\mathrm{L}$ & $\mathrm{A}$ & $\mathrm{b}$ & $\mathrm{CIE} x$ & $\mathrm{CIE} y$ & $\mathrm{DE}$ \\
\hline 0 & 100 & -60.0 & 16.1 & 0.297 & 0.356 & - \\
1 & 100 & -60.0 & 11.9 & 0.293 & 0.354 & 4.24 \\
2 & 100 & -60.0 & 9.7 & 0.292 & 0.353 & 6.42 \\
3 & 100 & -60.0 & 7.8 & 0.290 & 0.351 & 8.34 \\
4 & 100 & -60.0 & 6.9 & 0.289 & 0.351 & 9.26 \\
\hline
\end{tabular}

Table 4. Color Coordinates of the Transmission Spectra According to Time Exposure in Ammonia Atmosphere for BCP Film on Glass

\begin{tabular}{lcccccc}
\hline Exposure Time (min) & $\mathrm{L}$ & $\mathrm{A}$ & $\mathrm{b}$ & $\mathrm{CIE} x$ & $\mathrm{CIE} y$ & $\mathrm{DE}$ \\
\hline 0 & 100 & -14.4 & 57.0 & 0.351 & 0.357 & - \\
1 & 100 & -14.6 & 56.5 & 0.351 & 0.357 & 0.56 \\
2 & 100 & -14.8 & 55.0 & 0.350 & 0.357 & 1.98 \\
3 & 100 & -15.2 & 53.3 & 0.350 & 0.356 & 3.72 \\
4 & 100 & -15.4 & 51.4 & 0.349 & 0.355 & 5.68 \\
\hline
\end{tabular}

superior of 1 can be detected by the human eye. The resonant waveguide grating shows higher DE values in comparison to the BCP on glass. Thus it is possible to visually detect a color change after 1 minute of ammonia exposure (100 ppm) with the resonant grating.

\section{H. Considerations for a Divergent and Extended Light} Source in a Real Scenario

In the experimental setup presented above, a combination of a halogen light source, an optical fiber, and a microscope objective is used to form a collimated beam. However, in the real scenario the source could be divergent with an extended size as depicted in Fig. 14. Depending on the distance between the light source and the sensor, we can estimate the deviation of the reflected spectrum from an ideal collimated light source.

The deviation is related to the variation of incident angles, which can be calculated through the following relation:

$$
\begin{aligned}
d \times \sin \alpha & =\left[d \pm\left(\frac{w}{2}\right)\right] \times \sin \alpha^{\prime} \\
\sin \alpha^{\prime} & =\frac{d}{\left[d \pm\left(\frac{w}{2}\right)\right]} \sin \alpha .
\end{aligned}
$$

As an example we take the case where the light source has a width of $20 \mathrm{~cm}$ and is situated at a distance of $1 \mathrm{~m}$ from the sensor. The central incident beam angle is $17^{\circ}$. Inserting these values to the Eq. (18) we find:

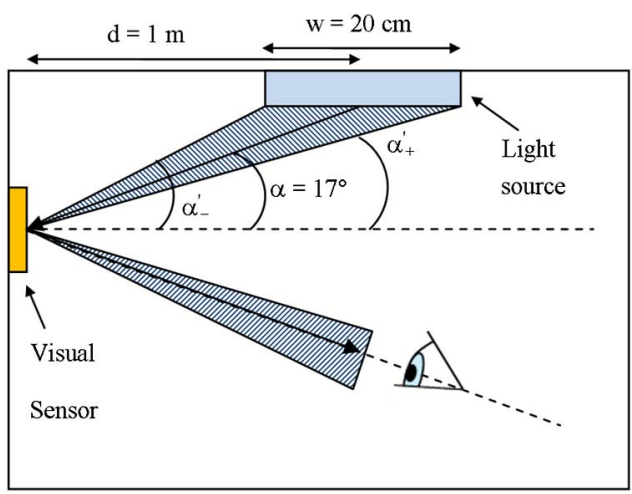

Fig. 14. (Color online) Sketch of a possible real scenario where the light source is not collimated. 


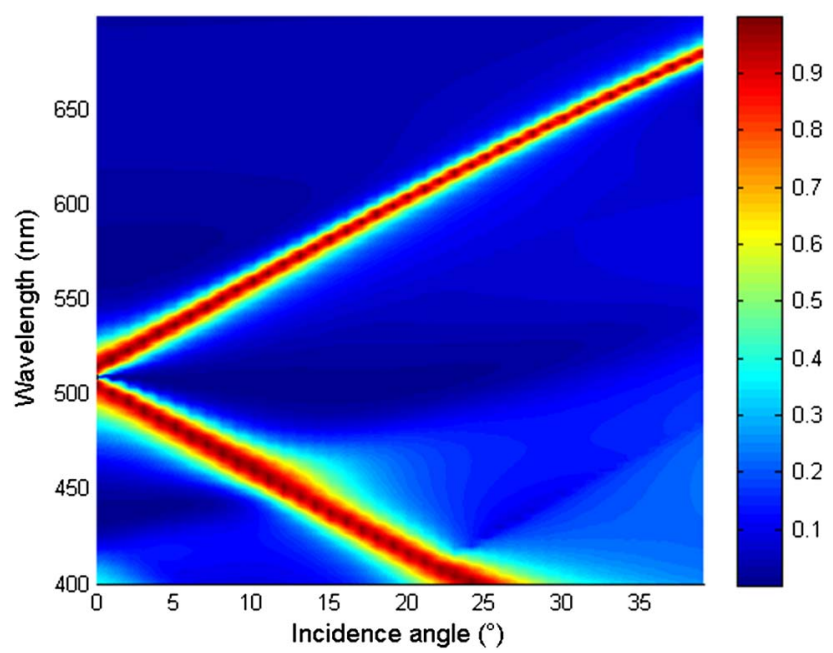

Fig. 15. (Color online) Reflection spectrum of the optimized structure according to incidence angle.

$$
\alpha_{-}^{\prime}=15.4^{\circ} \quad \text { and } \quad \alpha_{\mp}^{\prime}=18.9^{\circ} .
$$

Therefore the incident light angles to consider are $17^{\circ} \pm 2^{\circ}$. Figure 15 represents the reflected spectrum for different incident angles calculated for the optimized structure (Table 2). It shows a linear shift of resonant peaks wavelengths according to the incidence angle. The peak shift is about $4.5 \mathrm{~nm}$ for a $1^{\circ}$ angle shift.

In order to see the effect on the final color seen by the naked eye, we calculate the DE values according to the angle deviation (Table 5). The color coordinates are calculated by taking the average reflection spectra over the incidence angles considered. Resonant peaks get broader as the range of incident angles increases, as shown in Fig. 16.

Table $\underline{5}$ tells us that a color deviation can be seen when the variation of incidence angle is $1^{\circ}$ from the central angle. However, what matters in the present application is the color change seen when the device is exposed to ammonia. Therefore we calculate the deviation of color change when absorption coefficient $(k=0.01)$ is added to the BCP layer (Table $\underline{6}$ ).

We can see that the expected color change is constant over the angle deviation due to the symmetric distribution of resonant peaks from the central incidence angle. However if we increase further the incidence angle deviation, resonant peaks will be out of the absorption band (530-630 nm) of the BCP film. Since a $50 \mathrm{~nm}$ shift corresponds to a $10^{\circ}$ angle shift, no detection of color change by the naked eye can be expected for deviation angles larger than $\pm 10^{\circ}$.

Table 5. Color Coordinates and Color Deviation According to the Incidence Angle Deviation

\begin{tabular}{lcccccc}
\hline Angle $\left(^{\circ}\right)$ & $\mathrm{L}$ & $\mathrm{a}$ & $\mathrm{b}$ & $\mathrm{CIE} x$ & $\mathrm{CIE} y$ & $\mathrm{DE}$ \\
\hline $17^{\circ}$ & 46.5 & 43.3 & -40.9 & 0.289 & 0.195 & - \\
$A v \pm 0.5^{\circ}$ & 46.5 & 43.2 & -40.8 & 0.290 & 0.196 & 0.13 \\
$A v \pm 1^{\circ}$ & 46.5 & 42.9 & -40.6 & 0.290 & 0.196 & 0.52 \\
$A v \pm 2^{\circ}$ & 46.5 & 42.0 & -40.1 & 0.290 & 0.199 & 1.55 \\
\hline
\end{tabular}

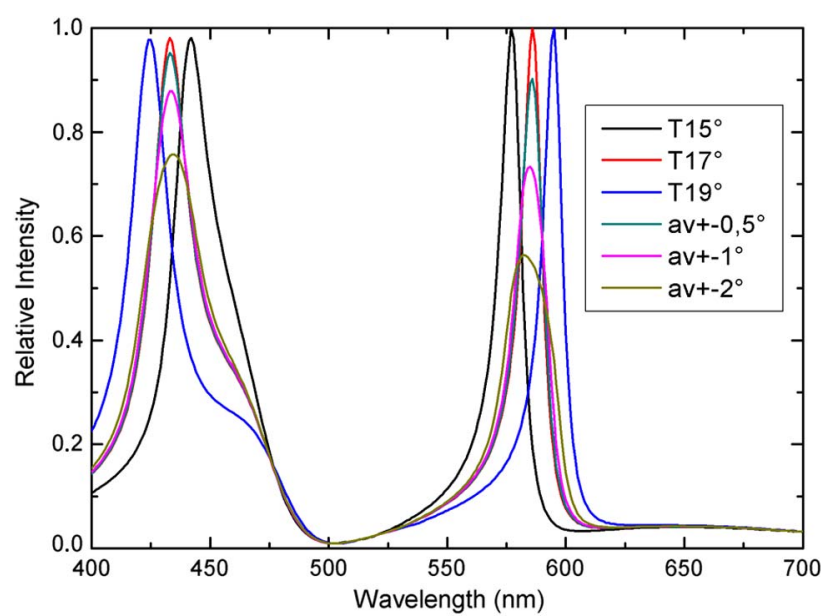

Fig. 16. (Color online) Average reflected spectrum according to the deviation angle.

Table 6. Color Coordinates and Color Changes with Absorption Coefficient $(k=0.01)$ Added to the BCP layer According to the Incidence Angle Deviation

\begin{tabular}{lcccccc}
\hline Angle $\left(^{\circ}\right)$ & $\mathrm{L}$ & $\mathrm{a}$ & $\mathrm{b}$ & $\mathrm{CIE} x$ & $\mathrm{CIE} y$ & $\mathrm{DE}$ \\
\hline $17^{\circ}$ & 42.9 & 41.4 & -41.9 & 0.280 & 0.187 & 4.2 \\
$A v \pm 0.5^{\circ}$ & 42.9 & 41.3 & -41.8 & 0.280 & 0.188 & 4.2 \\
$A v \pm 1^{\circ}$ & 42.9 & 41.0 & -41.6 & 0.280 & 0.189 & 4.2 \\
$A v \pm 2^{\circ}$ & 42.9 & 40.1 & -41.1 & 0.280 & 0.190 & 4.2 \\
\hline
\end{tabular}

\section{Conclusion}

We have demonstrated that visual chemical sensors based on subwavelength grating structures are feasible. An absorption model is developed to predict the resonant absorption conditions. It is validated with rigorous theoretical simulations. Optimization routines based on rigorous simulations have been implemented to select the highest photonic structure sensitivity in the case of ammonia detection with a thin BCP layer. Enhancement of the light absorption by the waveguide grating is shown experimentally with a fabricated sample. DE values have been extracted from the reflected spectra to evaluate the color changes in the case of an ideal incident collimated light. Light source divergence with an extended size is also considered to approach the real scenario configuration. Ammonia sensing tests have shown that the waveguide grating structure brings higher color changes (DE values).

One of its main advantages lies in its simple adjustability to spectral requirements. By changing the geometry of the resonant structures, the same dye and chemistry can be used to obtain different color shifts and different sensitivities. Different dyes can be integrated into the same platform to monitor different chemical reactions at the same time. The technology proposed here is fully compatible with mass production techniques, making the device a low cost-sensor. Furthermore, the device is a stand-alone sensor as any ambient light can be used as the light source (within certain conditions) and no external 
electrical supply is required. The main target applications concern the monitoring of gas contamination in public places in the case of terrorist attack or industrial accident, the monitoring of air pollution in cities, and the detection of gas leakages in private places.

Improvement of the current design could include a reference area built within the sensor to enhance the contrast of color changes.

\section{References}

1. S. S. Wang, R. Magnusson, J. S. Bagby, and M. G. Moharam, "Guided mode resonances in planar dielectric layer diffraction gratings," J. Opt. Soc. Am. A 7, 1470-1474 (1990).

2. D. Rosenblatt, A. Sharon, and A. A. Friesem, "Resonant grating waveguide structures," IEEE J. Quantum Electron. 33, 2038-2059 (1997).

3. M. T. Gale, K. Knop, and R. H. Morf, "Zero-order diffractive microstructures for security applications," Proc. SPIE 1210, 83-89 (1990).

4. S. S. Wang and R. Magnusson, "Theory and applications of guided mode resonance filters," Opt. Express 32, 2606-2613 (1993).

5. Y. Ding and R. Magnusson, "Resonant leaky-mode spectralband engineering and device applications," Opt. Express 12, 5661-5674 (2004).

6. B. Cunningham, P. Li, B. Lin, and J. Pepper, "Colorimetric resonant reflection as a direct biochemical assay technique," Sens. Actuators B 81, 316-328 (2002).

7. J. J. Wang, L. Chen, S. Kwan, F. Liu, and X. Deng, "Resonant grating filters as refractive index sensors for chemical and biological detections," J. Vac. Sci. Technol. B 23, 3006-3010 (2005).

8. J.-N. Yih, Y.-M. Chu, Y.-C. Mao, W.-H. Wang, F.-C. Chien, C.-Y. Lin, K.-L. Lee, P.-K. Wei, and S.-J. Chen, "Optical waveguide biosensors constructed with subwavelength gratings," Appl. Opt. 45, 1938-1942 (2006).

9. I. D. Block, N. Ganesh, M. Lu, and B. T. Cunningham, "A sensitivity model for predicting Photonic Crystal Biosensor Performance," IEEE Sens. J. 8, 274-280 (2008).

10. A. Sharon, S. Glasberg, D. Rosenblatt, and A. A. Friesem, "Metal-based resonant grating waveguide structures," J. Opt. Soc. Am. A 14, 588-595 (1997).

11. O. Stenzel, "Resonant reflection and absorption in grating waveguide structures," Proc. SPIE 5355, 1-13 (2004).

12. A. Greenwell, S. Boonruang, and M. G. Moharam, "Effect of loss or gain on guided mode resonant devices," in Integrated Photonics Research and Applications/Nanophotonics, OSA Technical Digest (Optical Society of America, 2006), paper NThA1.

13. Y. Park, E. Drouard, O. El Daif, X. Letartre, P. Viktorovitch, A. Fave, A. Kaminski, M. Lemiti, and C. Seassal, "Absorption enhancement using photonic crystals for thin silicon solar cells," Opt. Express 17, 14312-14321 (2009).

14. S. T. Peng, T. Tamir, and H. L. Bertoni, "Theory of periodic dielectric waveguides," IEEE Trans. Microwave Theor. Tech. MTT-23, 123-133 (1975).

15. L. Li, "Reformulation of the fourier modal method for surfacerelief gratings made with anisotropic materials," J. Mod. Opt. 45, 1313-1334 (1998).

16. D. Ashlock, Evolutionary Computation for Modeling and Optimization (Springer, 2006).

17. S. S. Sarkisov, D. E. Diggs, G. Adamovsky, and M. J. Curley, "Single-arm double-mode double-order planar waveguide interferometric sensor," Appl. Opt. 40, 349-359 (2001).

18. M. T. Gale, C. Gimkiewicz, S. Obi, M. Schnieper, J. Söchtig, H. Thiele, and S. Westenhöfer, "Replication technology for optical microsystems," Opt. Lasers Eng. 43, 373-386 (2005).

19. M. T. Gale, "Replication technology for micro optics and optical microsystems," Proc. SPIE 5177, 113-120 (2003). 